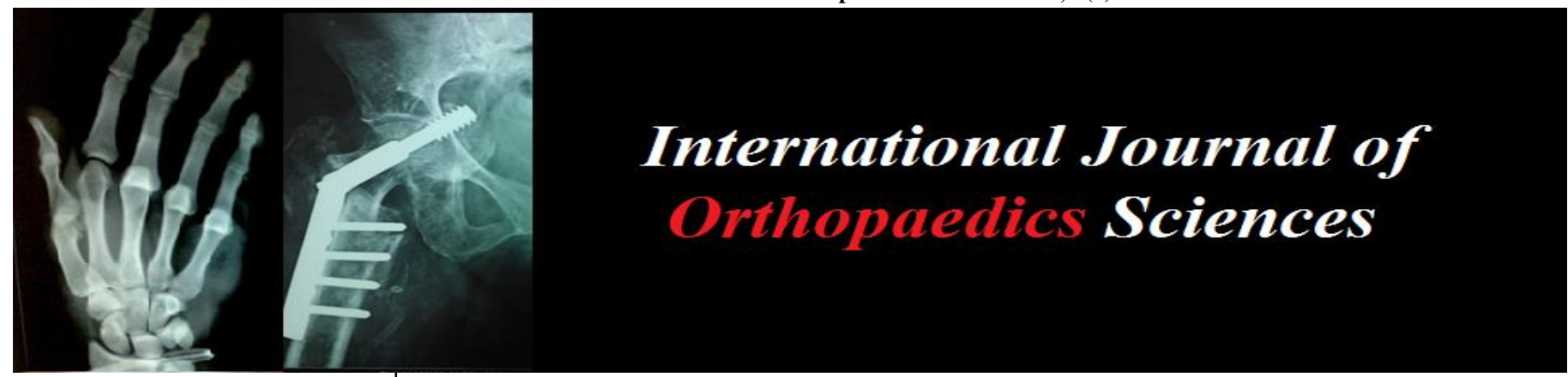

ISSN: $2395-1958$

IJOS 2019; 5(3): 138-140

(C) 2019 IJOS

www.orthopaper.com

Received: 10-05-2019

Accepted: 12-06-2019

Dr. BS Vijaya Kumar

Associate Professor, Vydehi

Institute of Medical Science and

Research Centre, Bengaluru,

Karnataka, India
Correspondence

Dr. BS Vijaya Kumar

Associate Professor, Vydehi

Institute of Medical Science and

Research Centre, Bengaluru,

Karnataka, India

\section{Surgical management of proximal humeral fractures by locking plates}

\section{Dr. BS Vijaya Kumar}

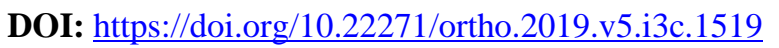

\section{Abstract}

A humerus fracture is a break of the humerus bone in the upper arm. Symptoms may include pain, swelling, and bruising. There may be a decreased ability to move the arm and the person may present holding there elbow. Complications may include injury to an artery or nerve, and compartment syndrome.

Objective: To study the efficacy, functional outcome for union of the fracture following surgery with locking plates in proximal humeral fractures. The present study was carried out between June 2015 to May 2016 in Orthopedic Department, Vydehi institute of medical sciences and research centre. In the proposed study 30 cases presenting with proximal humeral fractures are evaluated clinically and radio logically. In the present study maximum number of patients belongs to 31 to 40 years age group (12 cases) followed by 41 to 50 years age group ( 8 cases), maximum number of patients sustained femur fracture due to RTA ( 22 cases) followed by fall ( 08 cases), maximum number of patients sustained two part fracture (17 cases), followed by three part fracture (09 cases), four part fracture was seen in 3 cases and fracture dislocation in 1 case, 10 patients had excellent functional results and 15 patients had good functional outcome, while only 4 patients had fair functional outcome. The majority of patients can be treated with a sling or brace, and with time the fracture will heal. Casting is not possible with most types of humerus fractures. Surgery may be required when the bone fragments are far out of position.

Keywords: Surgical management, proximal humeral fractures, locking plates

\section{Introduction}

A humerus fracture is a break of the humerus bone in the upper arm. Symptoms may include pain, swelling, and bruising. There may be a decreased ability to move the arm and the person may present holding there elbow. Complications may include injury to an artery or nerve, and compartment syndrome ${ }^{[1,2]}$.

The cause of a humerus fracture is usually physical trauma such as a fall. Other causes include conditions such as cancer in the bone. Types include proximal humeral fractures, humeral shaft fractures, and distal humeral fractures. Diagnosis is generally confirmed by X-rays. A CT scan may be done in proximal fractures to gather further details.

Treatment options may include a sling, splint, brace, or surgery. In proximal fractures that remain well aligned, a sling is often sufficient. Many humerus shaft fractures may be treated with a brace rather than surgery. Surgical options may include open reduction and internal fixation, closed reduction and percutaneous pinning, and intramedullary nailing. Joint replacement may be another option. Proximal and shaft fractures generally have a good outcome well outcomes with distal fractures can be less good. They represent about $4 \%$ of fractures.

Proximal humerus fractures most often occur among elderly people with osteoporosis who fall on an outstretched arm. Less frequently, proximal fractures occur from motor vehicle accidents, gunshots, and violent muscle contractions from an electric shock or seizure. Other risk factors for proximal fractures include having a low bone mineral density, having impaired vision and balance, and tobacco smoking. A stress fracture of the proximal and shaft regions can occur after an excessive amount of throwing, such as pitching in baseball. ${ }^{[5,6]}$

Middle fractures are usually caused by either physical trauma or falls. Physical trauma to the humerus shaft tends to produce transverse fractures whereas falls tend to produce spiral fractures. Metastatic breast cancer may also cause fractures in the humerus shaft. 
Long spiral fractures of the shaft that are present in children may indicate physical abuse.

Distal humerus fractures usually occur as a result of physical trauma to the elbow region. If the elbow is bent during the trauma, then the olecranon is driven upward, producing a Tor Y-shaped fracture or displacing one of the condyles.

Humerus fractures are among the most common of fractures. Proximal fractures make up $5 \%$ of all fractures and $25 \%$ of humerus fractures, middle fractures about $60 \%$ of humerus fractures (12\% of all fractures), and distal fractures the remainder. Among proximal fractures, $80 \%$ are one-part, $10 \%$ are two-part, and the remaining $10 \%$ are three- and fourpart. The most common location of proximal fractures is at the surgical neck of the humerus. Incidence of proximal fractures increases with age, with about $75 \%$ of cases occurring among people over the age of 60 . In this age group, about three times as many women than men experience a proximal fracture. Middle fractures are also common among the elderly, but they frequently occur among physically active young adult men who experience physical trauma to the humerus. Distal fractures are rare among adults, occurring primarily in children who experience physical trauma to the elbow region.

\section{Objective}

To study the efficacy, functional outcome for union of the fracture following surgery with locking plates in proximal humeral fractures.

\section{Methodology}

The present study was carried out between June 2015 to May 2016 in Orthopedic Department, Vydehi institute of medical sciences and research centre. In the proposed study 30 cases presenting with proximal humeral fractures are evaluated clinically and radio logically.

\section{Inclusion Criteria}

- Age group: $>18$ years

- Gender: Male and female patients

- Two part, three part and four part fracture of proximal humerus

\section{Exclusion Criteria}

- Children and adolescent patients $<18$ yrs

- Compound fractures

- Undisplaced fractures

\section{Results}

Table 1: Age and Sex wise distribution of cases

\begin{tabular}{|c|c|c|c|}
\hline Age & Male & Female & Total \\
\hline $20-30$ & 02 & 00 & 03 \\
\hline $31-40$ & 07 & 05 & 12 \\
\hline $41-50$ & 06 & 02 & 08 \\
\hline $51-60$ & 03 & 01 & 04 \\
\hline $61-70$ & 02 & 02 & 04 \\
\hline Total & 20 & 10 & 30 \\
\hline
\end{tabular}

In the present study maximum number of patients belongs to 31 to 40 years age group ( 12 cases) followed by 41 to 50 years age group ( 8 cases)

Table 2: Distribution of Cases based on Mode of Injury

\begin{tabular}{|c|c|}
\hline Mode of injury & Number of patients \\
\hline RTA & 22 \\
\hline FALL & 08 \\
\hline Total & 30 \\
\hline
\end{tabular}

In the present study maximum number of patients sustained femur fracture due to RTA ( 22 cases) followed by fall ( 08 cases).

Table 3: Distribution of Cases based on Type of Fracture:

\begin{tabular}{|c|c|}
\hline Type of fracture & Number of patients \\
\hline Two Part & 17 \\
\hline Three Part & 09 \\
\hline Four Part & 03 \\
\hline Fracture Dislocation & 01 \\
\hline Total & 30 \\
\hline
\end{tabular}

In the present study maximum number of patients sustained two part fracture (17 cases), followed by three part fracture ( 09 cases), four part fracture was seen in 3 cases and fracture dislocation in 1 case.

Table 4: Distribution of Cases based on Outcome:

\begin{tabular}{|c|c|}
\hline Functional outcome & Number of patients \\
\hline Excellent & 10 cases \\
\hline Good & 15 cases \\
\hline Fair & 04 cases \\
\hline Poor & 01 cases \\
\hline Total & 30 \\
\hline
\end{tabular}

In our study 10 patients had excellent functional results and 15 patients had good functional outcome, while only 4 patients had fair functional outcome.

\section{Discussion}

In the present study maximum number of patients belongs to 31 to 40 years age group (12 cases) followed by 41 to 50 years age group ( 8 cases), maximum number of patients sustained femur fracture due to RTA ( 22 cases) followed by fall (08 cases), maximum number of patients sustained two part fracture (17 cases), followed by three part fracture (09 cases), four part fracture was seen in 3 cases and fracture dislocation in 1 case, 10 patients had excellent functional results and 15 patients had good functional outcome, while only 4 patients had fair functional outcome.

In 2009 AA Martinez et al., ${ }^{[5]}$ retrospectively reviewed 58 patients who underwent locking compression plate fixation for proximal humerus fracture between September 2004 to march 2006. all fractures healed satisfactorily, expect in 1 patient with a valgus 4-part fracture who had malunion. Functional outcome was excellent in 13 patients; good in 36, moderate in 8 , poor in 1 they concluded proximal humerus locking compression plate is appropriate treatment for proximal humerus fracture.

In 2009 MA Fazal et al., [6] retrospectively reviewed 27 patients who underwent locking compression plate fixation for proximal humerus fracture between June 2003 to June 2006. All fracture were classified as 2 part $(n=13), 3$ part $(n=12), 4$ part $(n=2)$.all fractures united expect one 3-part fracture in $78 y$ rs aged women in whom there was a collapse and screw penetration. Mean constant shoulder score was 70.11 patients had score exceeding 75, 13 were scored 
between 50 and 75, and 3 were below 50.They concluded philos plate fixation provided stable fixation, minimal metal work problem and enabled early range of motion exercises to achieve acceptable functional results.

In 2010 Sameer Aggarwal et al., ${ }^{[7]}$ over two and a half years, treated 56 patients with an acute proximal humerus fracture with locking plate osteosynthesis. 47 of these patients who completed a minimum follow up of 1 year were evaluated using Constant score calculation. The average follow up period was around 21.5 months. Outcomes were excellent in $17 \%$, good in $38.5 \%$, moderate in $34 \%$ while poor in $10.5 \%$. The Constant score was poorer for AO-OTA type 3 fractures as compared to other types. The scores were also inferior for older patients (> 65 years old). Complications included screw perforation of head, AVN, subacromial impingement, loss of fixation, axillary nerve palsy and infection. A varus malalignment was found to be a strong predictor of loss of fixation. They concluded locking plate osteosynthesis leads to satisfactory functional outcomes in all the patients. Results are better than non locking plates in osteoporotic fractures of the elderly.

In 2011 Georg Osterhoff, Ossendorf et al., ${ }^{[8]}$ studied patients with proximal humerus fracture who underwent angular stable plate fixation between 2007 to 2009. follow up was possible in 60 patients. Patients with calcar screw were assigned to group $\mathrm{c}+$, patients without calcar screw to group c-. Humeral head necrosis occurred in 6 patients in $(\mathrm{c}+, 15.4 \%)$ and 3 in (c-, $14.3 \%)$. Cut out of the proximal screw were observed in 3(c+, $7.7 \%)$ and 1 (c-, $4.8 \%)$ cases. In each group 1 patient showed delayed union. There was significant loss of reduction in group c- compared to $\mathrm{c}+$. He concluded the placement of calcar screw in angular stable plate fixation of proximal humerus fracture is associated with less secondary loss of reduction.

In 2012 Adithya C Pawaskar, Kee-Won lee et al., ${ }^{[9]}$ studied 25 patients who underwent surgery for proximal humerus fracture with locking plate between 2008 and 2010. Measurement of neck shaft angle was done at immediate post op, 3 month post op and final follow up (8 to 17 months). He found mean loss in neck shaft angle in the first 3 month was $3.8^{\circ}$ as compared to $1.3^{\circ}$ in the p period between 3 months and final follow up. This was statistically significant $(\mathrm{p}=0.002)$. He concluded proximal humerus locking plate maintains reliable radiographic results even in elderly population with proximal humerus fracture.

\section{Conclusion}

The majority of patients can be treated with a sling or brace, and with time the fracture will heal. Casting is not possible with most types of humerus fractures. Surgery may be required when the bone fragments are far out of position. Determining when the alignment is acceptable depends on a number of factors. Fractures close to the shoulder and elbow joints, especially fractures that extend into the joint, are more likely to require surgery. Conversely, fractures in the center of the shaft of the bone rarely require surgery, even with the bone fragments appear not perfectly aligned.

\section{References}

1. Auth PC. Physician Assistant Review. Lippincott Williams \& Wilkins, 2012, 167. ISBN 9781451171297.

2. Malhotra R. Mastering Orthopedic Techniques: Intraarticular Fractures. Jaypee Brothers Medical Publishers Pvt. Ltd, 2013, 47-62. ISBN 9789350908266.

3. Cameron P, Jelinek G, Kelly AM, Brown AFT, Little M.
Textbook of Adult Emergency Medicine. Elsevier Health Sciences, 2014, 167-170. ISBN 9780702054389.

4. Crosby L, Neviaser R. Proximal Humerus Fractures: Evaluation and Management. Springer, 2014, ISBN 9783319089515.

5. Martinez AA, et al. proximal humerus locking plate for proximal humerus fracture -retrospective study journal of orthopaedic surgery. 2009; 17(1):10-4.

6. Fazal MA, Haddad FS. PHILOS plate fixation for displ aced proximal humeral fractures journal of orthopaedic surgery. 2009; 17(1):15-8.

7. Sameer A et al. Displaced proximal humeral fractures: an Indian experience with locking plate. Journal of Orthopaedic surgery and research. 2010; 5:60.

8. Georg $\mathrm{O}$, Christian $\mathrm{O}$ et al. The calcar screw in angular stable plate fixation of proximal humerus fractures-a case study. Journal of orthopedic surgery and research. 2011; 6:50.

9. Adithya CP, Kee-Won Lee. Locking plate for proximal humerus fracture in the elderly population: serial change in neck shaft angle clin orthop surg. 2012; 4(3):209-215. 